\title{
One-Year Outcome of Small-Vessel Disease Treated with Sirolimus-Eluting Stents: A Subgroup Analysis of the e-SELECT Registry
}

\author{
RUNLIN GAO, M.D., ${ }^{1}$ ALEXANDRE ABIZAID, M.D., ${ }^{2}$ ADRIAN BANNING, M.D., ${ }^{3}$ ANTONIO L. \\ BARTORELLI, M.D., ${ }^{4}$ VLADIMÍRD ŽAVÍK, M.D., ${ }^{5}$ STEPHEN ELLIS, M.D. ${ }^{6}$ MYUNG HO JEONG, M.D., ${ }^{7}$ \\ VICTOR LEGRAND, M.D., ${ }^{8}$ CHRISTIAN SPAULDING, M.D.,PH.D., ${ }^{9}$ and PHILIP URBAN, M.D., ${ }^{10}$ for the \\ e-SELECT Investigators
}

From the ${ }^{1}$ Cardiovascular Institute and Fu Wai Hospital, Beijing, China $;{ }^{2}$ Instituto Dante Pazzanese de Cardiologia, São Paolo, Brazil; ${ }^{3}$ John Radcliffe Hospital, Oxford, United Kingdom; ${ }^{4}$ Centro Cardiologico Monzino, IRCCS, University of Milan, Milan, Italy; ${ }^{5}$ Peter Munk Cardiac Centre, University Health Network, Toronto, Canada; ${ }^{6}$ Cleveland Clinic Foundation, Cleveland, Ohio; ${ }^{7}$ The Heart Center of Chonnam National University Hospital, GwangJu, Korea; ${ }^{8}$ Centre Hospitalier Universitaire, Liège, Belgium; ${ }^{9}$ Cardiology Department, European Hospital Georges Pompidou, Assistance Publique Hôpitaux de Paris and INSERM U 970, Paris Descartes University, Paris, France; and ${ }^{10}$ La Tour Hospital, Geneva, Switzerland

\begin{abstract}
Objectives: To investigate the characteristics and one-year outcomes following sirolimus-eluting CYPHER Select Plus stent (SES) implantation in small (SmVD) and non-small vessel disease (NSmVD) in the international e-SELECT registry.

Background: Large-scale registry data are lacking on DES outcomes in SmVD treatment.

Methods: There were 4,700 SmVD (at least one vessel with estimated reference vessel diameter [RVD] $<2.5 \mathrm{~mm}$, excluding 283 patients with unknown RVD vessels) and 10,139 NSmVD only patients.

Results: The SmVD population was older, with more women, diabetics, and vessels treated, higher mean Charlson Comorbidity Index score (CCI), shorter lesions, and less STEMI presentation. The 1-year stent thrombosis (ST) rate (primary end-point), was significantly higher $(1.3 \%$ vs. $0.7 \%)$ in SmVD versus NSmVD, mainly driven by early events. One-year major adverse cardiac event (MACE), myocardial infarction (MI), and clinically indicated target-lesion revascularization (TLR) rates were significantly higher in SmVD although death and major bleeding rates were similar in both groups. Complication rates were similar between pure (3,188 patients; only RVD < $2.5 \mathrm{~mm})$ and mixed (1,795 patients; some RVD $<2.5 \mathrm{~mm}$ or unknown RVD) SmVD. Multivariate predictors for 1-year MACE in SmVD included saphenous vein graft or bifurcation lesions, major bleeding, any antiplatelet therapy discontinuation within 1 month, age, number of stents implanted, CCI, acute coronary syndrome, and insulin-dependent diabetes mellitus.

Conclusion: SES implantation for SmVD occurs more frequently in women, diabetics, and those with multivessel disease and comorbidities. One-year ST, MACE, MI, and clinically indicated TLR rates are higher, although low overall, in SmVD or mixed SmVD patients while death rates are similar to NSmVD. (J Interven Cardiol 2013;26:163-172)
\end{abstract}

Dr. Urban has served as consultant for Cordis and Biosensors. Dr. Banning has received research funding from Boston Scientific and Cordis; and his salary is partially funded by the NIHR Oxford Biomedical Research Centre. Dr. Bartorelli has served as a consultant to Abbott Vascular and is on the Speakers' Bureaus of Bracco and Cordis, Johnson \& Johnson. Dr. Dzavik has served as consultant for Abbott Vascular and has received educational funds from
Cordis. Dr. Legrand has served as consultant for Cordis and is a member of the scientific advisory board of Abbott. Pr. Spaulding has received research funding from Cordis, Abbott, Stentys, and Lilly; has received speaker fees from Cordis, Lilly, and Pfizer; was on the scientific advisory board of Cordis; and has been a full-time employee of Cordis, Johnson and Johnson from July 2010 to December 2011. Dr Jeong has received research funding from Cordis, Abbott, Stentys, and Lilly; speaker fees from Cordis, Lilly, and Pfizer; 
GAO, ET AL.

\section{Introduction}

From approximately one-third to over one-half of percutaneous coronary intervention (PCI) is targeted at significant disease in small coronary artery segments, defined as those with estimated reference vessel diameter (RVD) under a threshold ranging from $2.5 \mathrm{~mm}$ to $3 \mathrm{~mm}$ depending on the study. ${ }^{1-5} \mathrm{PCI}$ of small coronary artery segments, relative to that of larger caliber vessels, is significantly and directly associated with an increased risk of major adverse cardiovascular events (MACE), including restenosis and stent thrombosis (ST) ${ }^{6-9}$ Some studies have reported higher rates of target vessel revascularization in small compared to large vessels even with comparable postprocedural minimal in-stent lumen areas, which could be a consequence of the greater proportions of higher risk baseline clinical and lesion characteristics, including diabetes, multivessel disease (MVD), diffuse disease, and chronic total occlusion, among patients with small-vessel disease (SmVD). ${ }^{1,10}$ The clinical relevance and management, including medical therapy, PCI, and coronary artery bypass graft surgery (CABG) of SmVD is influenced by lesion location, amount of myocardium at risk, and occlusion severity. ${ }^{11} \mathrm{CABG}$ for revascularization of small coronary vessels is limited by high rates of technical failure. ${ }^{12}$ For PCI of SmVD, various devices and techniques have been used over time; however, it was not until the introduction of drug-eluting stents (DES) that outcomes in this challenging higher-risk setting were greatly improved, ${ }^{1,2,13-25}$ likely because, compared with large vessels, small vessels have a smaller postprocedural luminal area that is less able to accommodate neointimal hyperplasia. ${ }^{25,26}$ Data on large "real-world" experiences with PCI of SmVD have been lacking and therefore this study is aimed at investigating the characteristics and 1-year outcomes and predictors of MACE following sirolimus-eluting CYPHER stent (SES, Cordis Corporation, Johnson and Johnson, Bridgewater, NJ, USA) implantation in SmVD relative to those in non-SmVD (NSmVD) in the international e-SELECT registry, which was conducted in 320 hospitals throughout 56 countries, recruited 15,147 patients,

and was on the scientific advisory board of Cordis. Dr Gao has received research supports from Abbott Vascular, Boston Scientific, Medtronic, B Braun, and MicroPort Medical (Shanghai, China).

Address for reprints: Runlin Gao, M.D., Cardiovascular Institute and Fu Wai Hospital, 167 Beilishi Road, Beijing 100037, China. Fax: 86-1068333879; e-mail: gaorunlin@citmd.com and has been the subject of 5 publications focused on different clinical subsets. ${ }^{27-31}$

\section{Methods}

The design, execution, and data analysis of the eSELECT registry has been previously described. ${ }^{27-31}$ The e-SELECT registry included 320 medical centers in 56 countries. Baseline data were collected between May 2006 and April 2008 in consecutive and eligible patients who underwent implantation of $\geq 1$ CYPHER Select Plus (Cordis Corporation, Johnson and Johnson, Bridgewater, NJ, USA) SES according to standard practice and procedural techniques. The protocol specified very few inclusion or exclusion criteria. Although lesions could be pretreated with any technique or device (such as balloon angioplasty, cutting balloon, or atherectomy), implantation of SES in each target lesion during index procedure was mandatory. All postoperative medical management, including antithrombotic therapy, was prescribed according to usual local practice. The protocol was approved by the ethics committee of each participating medical center, and the patients granted their consent to participate in the registry. Patients for whom the collection of dependable followup information was unlikely and those who received a stent other than a CYPHER SES during the index procedure were excluded. RVD was visually estimated by angiography, and small vessels were defined as those with an RVD $\leq 2.5 \mathrm{~mm}$. The primary end-point of the registry was a composite of definite and probable ST at 1 year of follow-up, as defined by the Academic Research Consortium. ${ }^{32}$ Secondary end-points at 1 year included rates of major bleeding (MB) according to the STEEPLE (Safety and efficacy of Enoxaparin in PCI) definition, ${ }^{33}$ cardiac and noncardiac death, myocardial infarction (MI), and MACE (defined as any death, MI, or target lesion revascularization [TLR]). Of 15,122 all-comer patients in the e-SELECT registry, treatment of SmVD, defined as at least one vessel with estimated RVD $\leq 2.5 \mathrm{~mm}$ and excluding cases with unknown RVD, was done in 4,700 patients $(31 \%$; 7,318 lesions; 8,443 stents), although treatment of NSmVD only was performed in 10,139 patients $(67 \% ; 12,204$ lesions; 14,508 stents); proportions of cases with multiple stents, approximately $19 \%$, and of overlapped stents among multiple-stent cases, approximately $87 \%$, were similar for both groups. There were 3,188 with pure SmVD (RVD $\leq 2.5 \mathrm{~mm}$ only) and 1,795 with 


\section{ONE-YEAR OUTCOME OF SMALL-VESSEL DISEASE TREATED WITH SIROLIMUS-ELUTING STENTS}

mixed SmVD (some vessel with RVD $\leq 2.5 \mathrm{~mm}$ or unknown RVD, the latter amounting to 283 patients). In the overall e-SELECT registry, $98.2 \%$ of patients were eligible for follow-up at 12 months. Of these $92.0 \%$ underwent follow-up at a mean follow-up of $370.4 \pm$ 124.7 days. As previously reported for the e-SELECT registry, at 1 year (the follow-up length), the reported compliance with dual antiplatelet therapy (DAPT) as recommended by the European Society of Cardiology guidelines was $86.3 \% .^{27}$

Statistical Analysis. For all patients, standard descriptive statistics were used for baseline, lesion, and procedural characteristics and for clinical results. Continuous variables are presented as mean \pm SD and compared using the Student's t-test, and categorical variables are presented as numbers and percentages and compared using the chi-square test. Cumulative rates of adverse clinical events were calculated with event-specific adjusted denominators, therefore all patients experiencing an event within 360 days or followed up for at least 330 days after index procedure contributed to the denominator. There was no censoring. MACE-free survival curves were constructed by the Kaplan-Meier life-table method, and compared by the log-rank test. Predictors of major adverse cardiac events were identified by univariate and multivariate analyses with Cox proportional hazards model. The following baseline covariates with $>15 \%$ missing values were excluded from analysis: creatine kinase-MB isoenzyme (CK-MB) or troponin levels greater than upper limit of normal preprocedure; hemoglobin level preprocedure; left ventricular ejection fraction (LVEF), maximum inflation pressure, and platelet count. Baseline demographic, angiographic, clinical, and procedural covariates were identified by univariate analysis as significantly correlated $(\mathrm{P}<$ $0.05)$ with MACE, namely Charlson Comorbidity Index (CCI), saphenous vein graft $(\mathrm{SVG})^{34}$ target lesion, $\mathrm{MB}$, age, number of stents implanted, total stent length, bifurcation lesion, in-stent restenosis (ISR) target lesion, any deviation from continuous DAPT up to 1-month follow-up, acute coronary syndrome (ACS), diabetes mellitus (DM), insulin-dependent DM (IDDM), MVD, maximum lesion length, index procedure-related ST, previous CABG, bypass graft lesion, chronic pulmonary disease, chronic Vitamin $\mathrm{K}$ antagonists (AVK) treatment, total number of lesions treated, major or minor bleeding, postdilation, moderate to severe renal disease, multisent-treated patient, diabetes with retinopathy, neuropathy or nephropa- thy, multilesion-treated patient, calcification, history of prior MI, ostial location, American College of Cardiology/American Heart Association (ACC/AHA) lesion morphology class B2 or C, unprotected left main target lesion, history of hyperlipidemia, and predilatation. Following the rule of 20 events per predictor, 13 of the most clinically meaningful univariate parameters with $\mathrm{P}<0.05$ (first 13 in the aforementioned univariate predictors) were included in the multivariate analysis. All statistical analyses were performed with SAS (version 9.1 or higher software, SAS Institute, Cary, NC, USA).

\section{Results}

As shown in Table 1, the SmVD patient population was older $(63.2$ years vs. 61.7 years, $\mathrm{P}<0.001)$, with a higher proportion of women $(28.4 \%$ vs. $22.9 \%$, $\mathrm{P}<0.001)$, diabetics $(34.1 \%$ vs. $28.6 \%, \mathrm{P}<0.001)$, and higher mean CCI $(1.1 \pm 1.4$ vs. $1.0 \pm 1.3$, P $<0.001)$ and had more vessels treated (1.3 vs. 1.1, $\mathrm{P}<0.001)$. Patients with SmVD presented less often with ST-segment Elevation Myocardial Infarction (STEMI) $(5.8 \%$ vs. $7.6 \%, \mathrm{P}<0.001)$, and had shorter lesions (19.1 mm vs. $20.8 \mathrm{~mm}, \mathrm{P}<0.001)$. The rate of ARC-defined "definite or probable" ST was significantly higher in the SmVD group $(1.3 \%$ vs. $0.7 \%$, $\mathrm{P}<0.001$ ), mainly driven by a higher incidence of early ( $0-30$ days) ST $(0.9 \%$ vs. $0.4 \%, P=0.002$; Fig. 1). The incidence of MACE (any death, MI, or TLR, $5.4 \%$ vs. $4.0 \%, \mathrm{P}<0.001)$, MI $(2.4 \%$ vs. $1.5 \%, \mathrm{P}<$ $0.001)$, and clinically indicated TLR ( $2.7 \%$ vs. $1.8 \%, P$ $<0.001$ ) was significantly higher at 1 year in patients with SmVD, respectively (Fig. 2). The MACE-free survival rate at 1 year was significantly lower in SmVD than that in NSmVD (Fig. 3). The incidence of death (1.7\% vs. $1.5 \%, \mathrm{P}=0.287)$ and $\mathrm{MB}(1.0 \%$ vs. $0.8 \%$, $\mathrm{P}=0.218$ ) was similar in both groups. There was a numerical trend of increasing MACE rate with decreasing RVD (Fig. 4). The pure SmVD group had a higher proportion of women $(30.6 \%$ vs. $23.5 \%, \mathrm{P}<0.001)$, with more prior PCI ( $33.1 \%$ vs. $30.1 \%, \mathrm{P}=0.028)$, higher mean CCI (1.2 vs. $1.1, \mathrm{P}=0.026)$, more STEMI ( $6.5 \%$ vs. $4.7 \%, \mathrm{P}=0.0119)$, fewer number of vessels or lesions treated (1.1 vs. $1.6, \mathrm{P}<0.001$, and 1.2 vs. 2.3 , $\mathrm{P}<0.001$, respectively), and smaller RVD $(2.4 \mathrm{~mm}$ vs. $2.8 \mathrm{~mm}, \mathrm{P}<0.001)$. However, the MACE-free survival rate at 1 year was not significantly different between the pure and mixed SmVD groups (Fig. 5); the MACE-free survival rate at 1 year in the mixed 
GAO, ET AL.

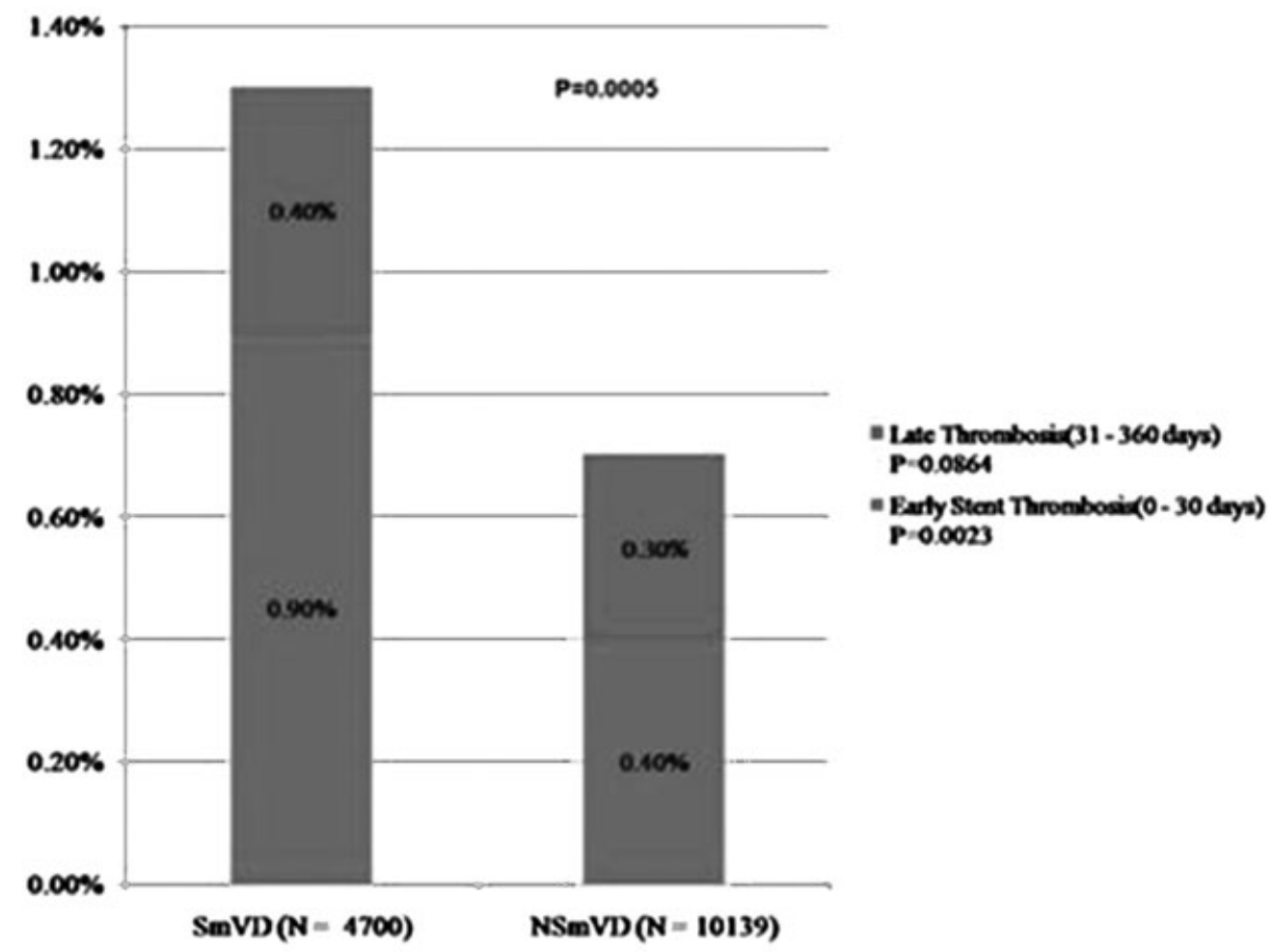

Figure 1. Histogram representation of definite/probable stent thrombosis according to the ARC definitions comparing the small-vessel disease and non-small-vessel disease subsets of the e-SELECT registry.

SmVD was significantly lower than that in NSmVD group (Fig. 6). The multivariate predictors for MACE in the SmVD group at 1 year included SVG or bifurcation target lesions, $\mathrm{MB}$, any deviation from continuous DAPT up to 1-month follow-up, age [years], number of total stents implanted, CCI, ACS, and IDDM; (Table 2). Stent overlap was not a univariate predictor for MACE at 1 year (Hazard Ratio (HR) 1.05, 95\% Confidence Interval (CI) 0.68-1.61, $\mathrm{P}=0.833)$.

\section{Discussion}

This study of one of the largest cohorts of SmVD ( $\leq 2.5 \mathrm{~mm}$ estimated RVD) confirms that SES implantation for SmVD occurs more frequently in women, diabetics, those with MVD, and comorbidities. Death rate at 1 year is similar to that of patients with NSmVD, and although incidence of MACE, MI, clinically indicated TLR, and ST is higher in patients with SmVD, and in those with mixed SmVD, it remains low overall. The proportion of SmVD treated in the e-SELECT registry, $31 \%$, is within the range of previously re- ported ones in real-world interventional cardiology studies. ${ }^{35}$ As further supported by this study, patients with smaller vessels have higher frequency of several characteristics, including DM and MVD that have been associated with a poorer outcome after stent implantation, ${ }^{1,36,38}$ smaller coronaries also are more common in certain groups of patients including women and Asians. ${ }^{19}$ The lower frequency of STEMI presentation in the SmVD versus NSmVD group (5.8\% vs. 7.6\%, $\mathrm{P}<0.001)$ stands in contrast to a previous report ${ }^{37}$ on a large cohort of 798 STEMI patients in whom approximately $50 \%$ of culprit lesions were located in smaller vessels $(<3 \mathrm{~mm})$. The increased risk of MACE in small relative to large vessels has been seen in previous studies $^{6-9}$ and in this study is mainly due to increased MI, ST, and TLR rates in SmVD patients, although the death rate is similar between the SmVD and NSmVD groups. Despite the remarkably low values for late lumen loss documented in DES studies, there remains a relationship between vessel size and restenosis, with increased restenosis rates in smaller vessels; the same is true for ST. Several studies, both randomized and nonrandomized, and subanalyses of all-comer studies have 
ONE-YEAR OUTCOME OF SMALL-VESSEL DISEASE TREATED WITH SIROLIMUS-ELUTING STENTS

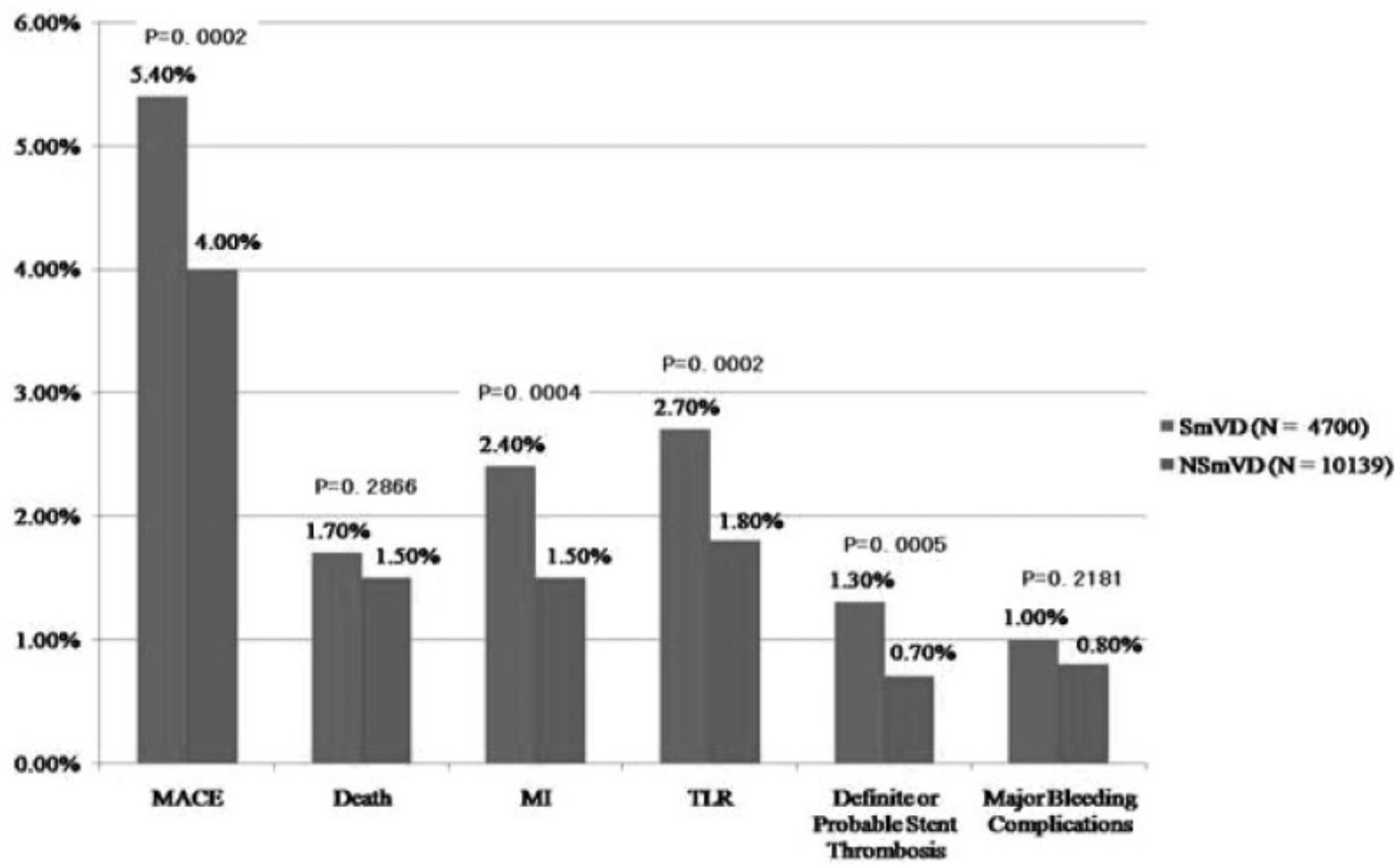

Figure 2. Major adverse clinical events at 1 year.

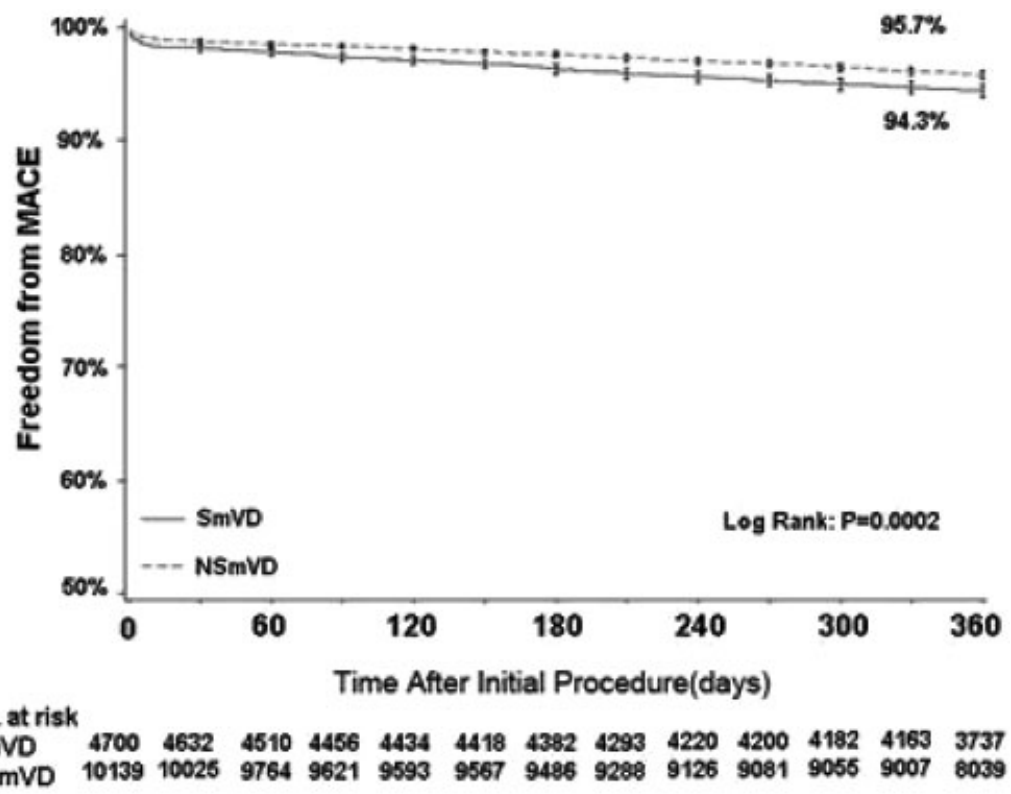

Figure 3. MACE-free survival curves at 1 year comparing patients with SmVD and NSmVD.
No. at risk

$\begin{array}{llllllllllllll}\text { SmVD } & 4700 & 4632 & 4510 & 4456 & 4434 & 4418 & 4382 & 4293 & 4220 & 4200 & 4182 & 4163 & 3737\end{array}$ NSmVD $1013910025976496219593 \quad 9567 \quad 94869288912690819056 \quad 9007 \quad 8039$ 
GAO, ET AL.

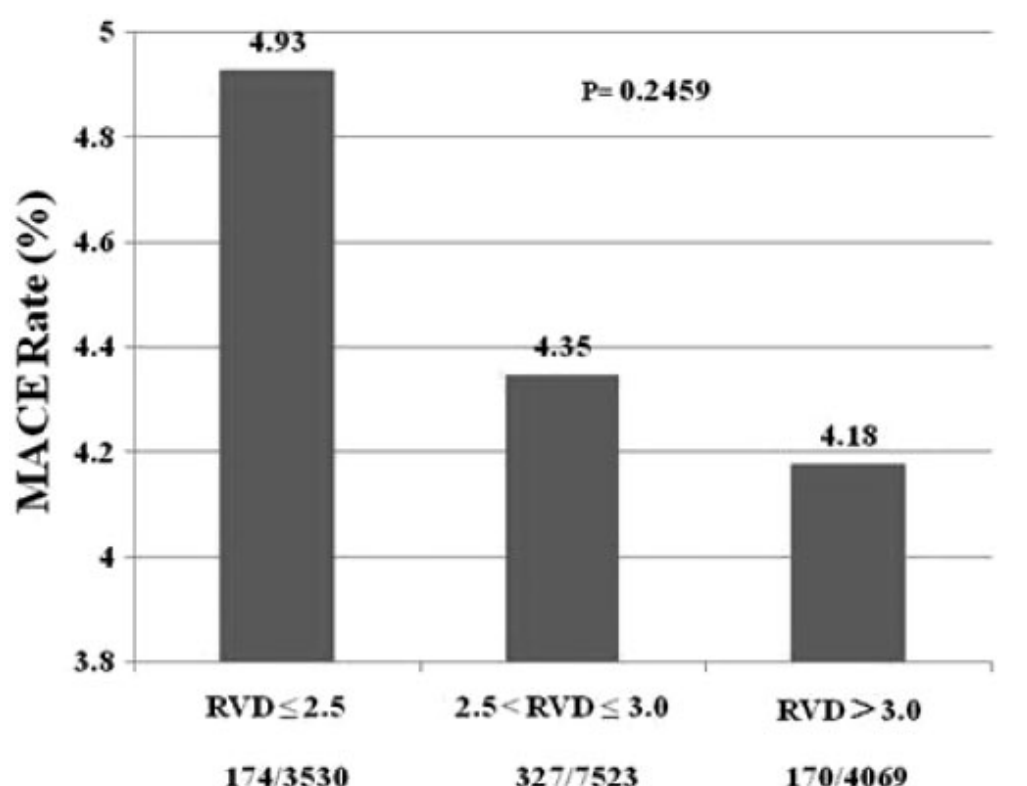

Figure 4. Correlation between MACE and RVD.

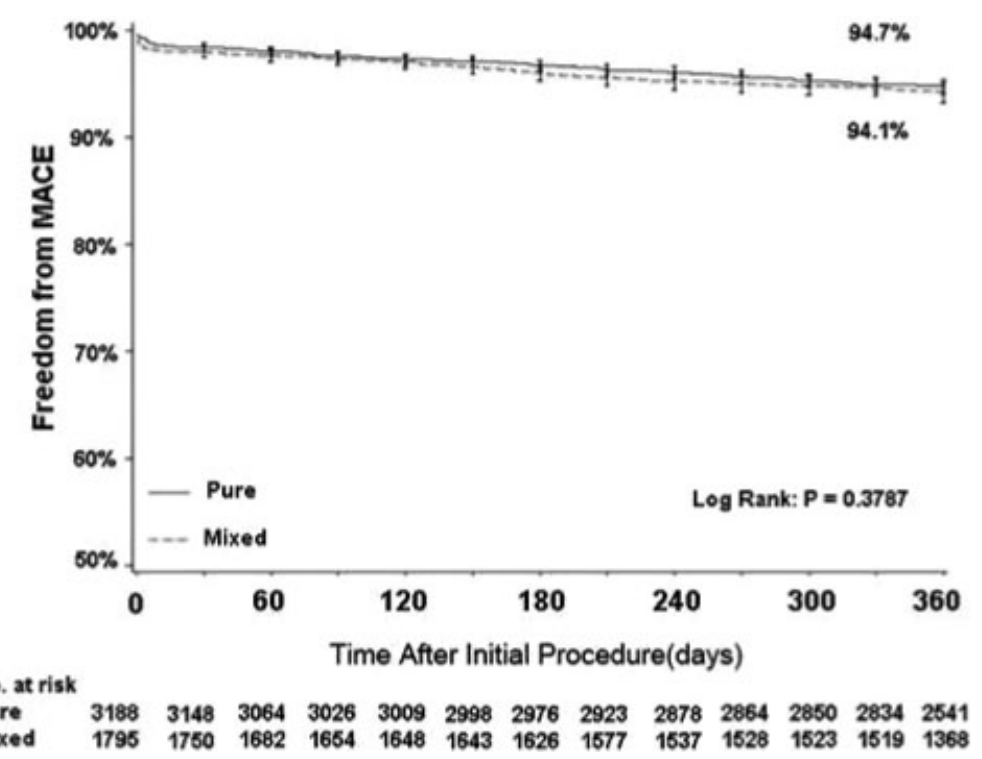

Figure 5. MACE-free survival curves at 1 year comparing patients with pure and mixed SmVD.

supported better outcomes in small vessels with SES, and in direct and indirect comparisons to PES. ${ }^{15,38-45}$ Although some interventionists support balloon-only angioplasty for SmVD treatment, ${ }^{46,47}$ SES has shown consistent benefit and lower rates of complications than alternative treatments ${ }^{12,38-40,42,48-51}$ even when using, as was not the case in this study, $2.5 \mathrm{~mm}$ stents in vessels with $<2.5 \mathrm{~mm}$ diameter, $40,44,52,53$ or in patients with diabetes and very small coronary vessel $(<2.1$ $\mathrm{mm}$ ) disease, ${ }^{15}$ or even relative to thinner-strut bare metal stents (BMS) or DES. ${ }^{17,54}$ In terms of the poten- tial for improving outcomes of PCI in SmVD, studies have shown that specific baseline clinical and angiographic characteristics are predictive of the different rates of MACE, including restenosis, seen for various lesion/patient subsets. ${ }^{39}$ Diabetes, for instance, exacerbates the negative impact on outcomes of smaller vessel size, ${ }^{39,55,56,57}$ and insulin treatment was a multivariate predictor of in-segment restenosis in the Taxus in Reallife Usage Evaluation (TRUE) registry. ${ }^{4}$ In this study IDDM was identified as one of the multivariate predictors of 1-year MACE. Hausleiter et al. ${ }^{55}$ and Iijima 
ONE-YEAR OUTCOME OF SMALL-VESSEL DISEASE TREATED WITH SIROLIMUS-ELUTING STENTS

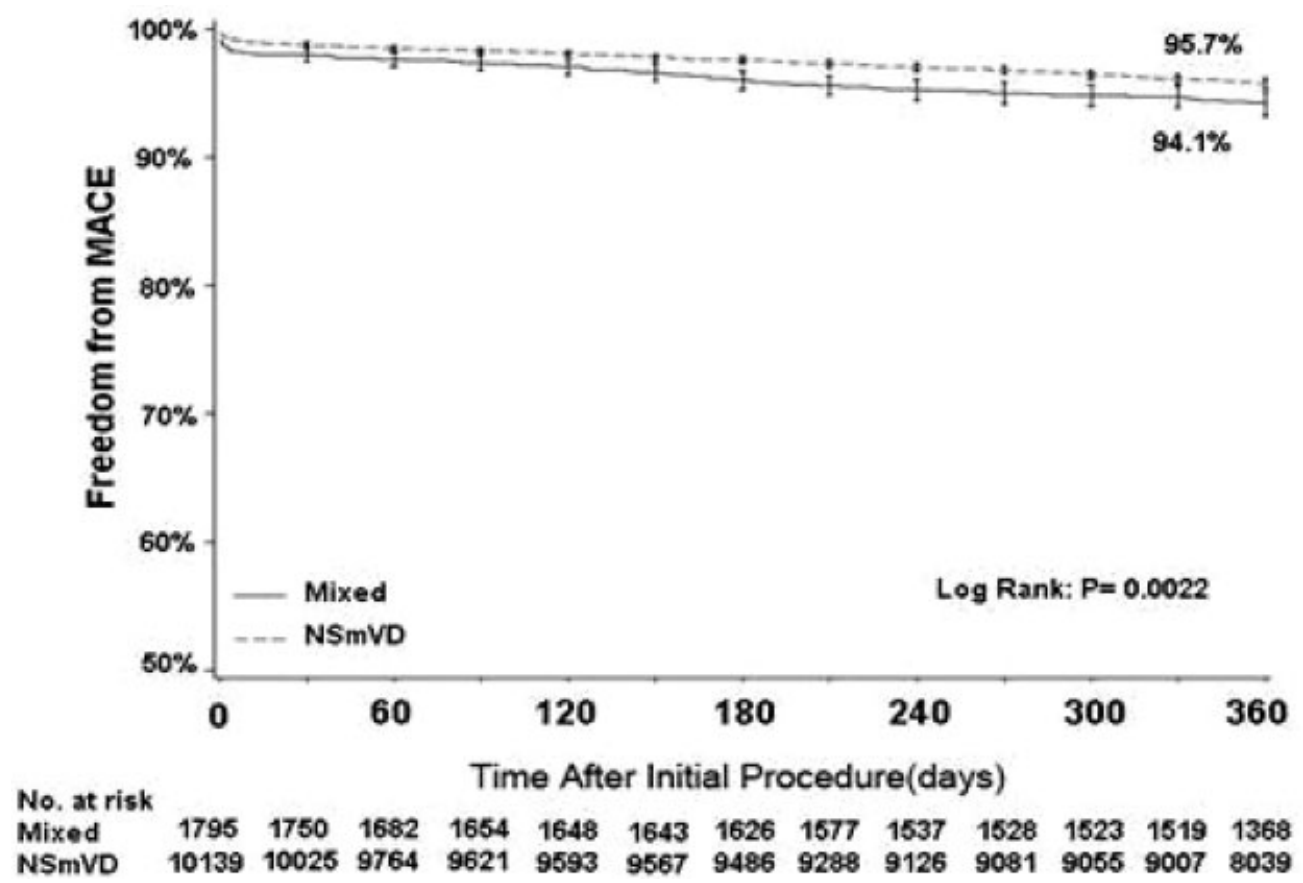

Figure 6. MACE-free survival curves at 1 year comparing patients with mixed SmVD and NSmVD.

Table 1. Baseline Patient and Procedural Characteristics of the SmVD and NSmVD Groups

\begin{tabular}{|c|c|c|c|}
\hline & $\operatorname{SmVD~N}=4,700$ & $\mathrm{NSmVD} N=10,139$ & P-value \\
\hline Age (years) & $63.2 \pm 11$ & $61.7 \pm 11$ & $<0.001$ \\
\hline Male (\%) & 71.6 & 77.1 & $<0.001$ \\
\hline Prior PCI $(\%)$ & 32.4 & 32.2 & 0.86 \\
\hline Prior CABG (\%) & 10.7 & 8.3 & $<0.001$ \\
\hline Prior MI (\%) & 31.6 & 32.6 & 0.23 \\
\hline Hypertension (\%) & 69.0 & 66.8 & 0.008 \\
\hline Hyperlipidemia (\%) & 70.8 & 67.3 & $<0.001$ \\
\hline History of smoking (\%) & 51.0 & 54.8 & $<0.001$ \\
\hline Diabetes mellitus (\%) & 34.1 & 28.6 & $<0.001$ \\
\hline Insulin treated DM (\%) & 29.2 & 25.8 & 0.015 \\
\hline $\mathrm{LVEF}<30 \%(\%)$ & 3.0 & 2.6 & 0.32 \\
\hline Charlson Comorbidity Index score & $1.1 \pm 1.4$ & $1.0 \pm 1.3$ & $<0.001$ \\
\hline STEMI $(\%)$ & 5.8 & 7.6 & $<0.001$ \\
\hline Number of vessels treated & $1.3 \pm 0.5$ & $1.1 \pm 0.4$ & $<0.001$ \\
\hline RVD (mm) & $2.6 \pm 0.4$ & $3.1 \pm 0.3$ & $<0.001$ \\
\hline Number of lesions treated & $1.6 \pm 0.8$ & $1.2 \pm 0.5$ & $<0.001$ \\
\hline Lesion length (mm) & $19.0 \pm 10.6$ & $20.8 \pm 12.0$ & $<0.001$ \\
\hline Number of stents/patient & $1.8 \pm 1.0$ & $1.4 \pm 0.7$ & $<0.001$ \\
\hline Number of stents/lesion & $1.16 \pm 0.42$ & $1.19 \pm 0.47$ & $<0.001$ \\
\hline Mean stent diameter (mm) & $2.6 \pm 0.2$ & $3.1 \pm 0.3$ & $<0.001$ \\
\hline Total stent length $(\mathrm{mm}) / \mathrm{lesion}$ & $24.2 \pm 12.1$ & $26.0 \pm 13.7$ & $<0.001$ \\
\hline Lesions with predilatation (\%) & 67.5 & 62.3 & $<0.001$ \\
\hline Stents with postdilatation (\%) & 33.1 & 37.8 & $<0.001$ \\
\hline DAPT postprocedure (\%) & 97.1 & 97.3 & 0.33 \\
\hline
\end{tabular}


GAO, ET AL.

Table 2. Multivariate Predictors of MACE at 1 Year in the SmVD Group

\begin{tabular}{lcr}
\hline Predictors of MACE to 360 days Multiple CoxPh regression & Hazard ratio [95\% CI] & P-value \\
\hline Charlson Comorbidity Index & $1.11[1.06-1.16]$ & $<0.001$ \\
SVG target lesion & $3.63[2.01-6.58]$ & $<0.001$ \\
Major bleeding & $3.35[1.69-6.63]$ & $<0.001$ \\
Age (years) & $1.02[1.01-1.03]$ & 0.002 \\
Number of stents implanted & $1.20[1.06-1.35]$ & 0.003 \\
Bifurcation lesion & $1.55[1.10-2.17]$ & 0.011 \\
Any deviation from continuous DAPT (up to 1-month follow-up) & $2.26[1.18-4.32]$ & $1.38[1.06-1.80]$ \\
ACS & $1.94[1.05-3.60]$ & 0.014 \\
Insulin-dependent diabetes mellitus & 0.034 \\
\hline
\end{tabular}

et al. ${ }^{57}$ identified total stent length as a predictor of restenosis in SmVD treated with BMS; however, stent length was not among multivariate predictors of insegment restenosis in a study of small vessel lesions $(<2.75 \mathrm{~mm})$ treated with PES. ${ }^{4}$ In a Korean study of 1,269 lesions in small coronaries $(\leq 2.8 \mathrm{~mm})$, lesion length was a powerful predictor of restenosis and MACE, with multiple overlapping stents in very long SmVD (lesion length $\geq 60 \mathrm{~mm}$ ) being associated with a high risk of SES failure. ${ }^{58}$ In this study total stent number but not total stent length or stent overlap was a multivariate MACE predictor in SmVD. The CCI score which captures cardiovascular status is a MACE predictor as reported by Hausleiter et al. ${ }^{55}$ which identified ACS at admission and LVEF as predictors of early adverse clinical outcomes in BMS-treated SmVD. Age was a MACE predictor of MACE in this and another report. ${ }^{10}$ As in this study, Iijima et al. ${ }^{57}$ identified bifurcation lesion as a MACE predictor. PCI of SVG is associated with worse outcomes and high incidence of ISR. ${ }^{5}$ The finding in this study that DAPT discontinuation during the first month postindex procedure is a predictor of 1-year MACE is consistent with previous reports ${ }^{59}$ and underscores the importance of DAPT use particularly during the first month postindex procedure; however, the finding that bleeding is also a MACE predictor in SmVD PCI calls for caution in the use of DES in the setting of patients at high risk for bleeding. This study showed that the MACE-free survival rate at 1 year was not significantly different between the pure and mixed SmVD groups; it was also significantly lower in mixed SmVD than in NSmVD which, to our knowledge, has not been previously reported in the literature. This finding has potential clinical significance. In clinical practice, patients with mixed vessel size (combined RVD $\leq 2.5 \mathrm{~mm}$ and RVD $>2.5 \mathrm{~mm}$ ) are common, and to avoid unnecessarily increasing MACE one needs to consider the severity of lesion stenosis and the territory of ischemia of the SmVD before treating the lesion.

This study is limited by the fact that the Cypher stent has been withdrawn from markets in most countries; however, SES are extensively used in many places around the world, and the lessons learned from this study may also be suitable for treatment of SmVD with other limus-eluting stents. The study was an international multicenter registry, and the inclusion and exclusion criteria nonetheless allow for an analysis with lessened confounding. Because of the extensive multinational and multicenter nature of the registry study, the RVD was visually estimated without quantitative coronary angiography analysis, which does not allow validation of the actual vessel sizes included in the groups studied. However, the vessel size threshold used is not uncommon and the majority of small vessels treated were of similar estimated RVD, namely 2.5 , as reflected by the standard deviation of 0.2. Also, although follow-up was conducted for 1 year, which does not address very late safety, i.e., events occurring beyond 1 year, the results of this international multicenter large-cohort study of outcomes after PCI of SmVD show favorable efficacy and safety of SES implantation in unselected patients and consistency with previous similar smaller studies. Only unadjusted rates of clinical events were compared between vessel size groups because the emphasis was on comparative epidemiology of SmVD and outcome predictors. Analyses also included pure and mixed SmVD groups which underscored the findings with the overall SmVD cohort (i.e., that including pure and mixed cases).

\section{Conclusion}

This large cohort study of SmVD confirms that SES implantation for SmVD occurs more frequently in women, diabetics, and those with MVD and 


\section{ONE-YEAR OUTCOME OF SMALL-VESSEL DISEASE TREATED WITH SIROLIMUS-ELUTING STENTS}

comorbidities. The incidence of MACE, MI, clinically indicated TLR, and ST is higher in patients with SmVD, whether pure or mixed SmVD, although it remains low overall. Death rate at 1 year is similar between SmVD and NSmVD. The multivariate predictors of 1-year MACE include SVG or bifurcation target lesions, MB, any deviation from continuous DAPT up to 1 month follow-up, age (years), number of total stents implanted, CCI, ACS, and IDDM.

Acknowledgment: The authors are grateful to Zhonglian $\mathrm{Li}$ and Amy Orlick for their contributions to this manuscript.

\section{References}

1. Biondi-Zoccai G, Moretti C, et al. Percutaneous coronary intervention for small vessel coronary artery disease. Cardiovasc Revasc Med 2010;11:189-198.

2. Bartorelli AL, Serruys PW, Miquel Hebert K, et al. An everolimus-eluting stent versus a paclitaxel-eluting stent in small vessel coronary artery disease: A pooled analysis from the SPIRIT II and SPIRIT III trials. Catheter Cardiovasc Interv 2010;76(1):60-66.

3. Wykrzykowska JJ, Serruys PW, Onuma Y, et al. Impact of vessel size on angiographic and clinical outcomes of revascularization with biolimus-eluting stent with biodegradable polymer and sirolimus-eluting stent with durable polymer the LEADERS trial substudy. JACC Cardiovasc Interv 2009;2(9):861870.

4. Godino C, Furuichi S, Latib A, et al. Clinical and angiographic follow-up of small vessel lesions treated with paclitaxel-eluting stents (from the TRUE registry). Am J Cardiol 2008;102:10021008.

5. Colombo A, Chieffo A. Drug-eluting stent update 2007: Part III: Technique and unapproved/unsettled indications (left main, bifurcations, chronic total occlusions, small vessels and long lesions, saphenous vein grafts, acute myocardial infarction, and multivessel disease). Circulation 2007;116:1424-1432.

6. Habara S, Mitsudo K, Goto T, et al. The impact of lesion length and vessel size on outcomes after sirolimus-eluting stent implantation for in-stent restenosis. Heart 2008;94(9):11621165.

7. Hsieh IC, Chien CC, Chang HJ, et al. Acute and long-term outcomes of stenting in coronary vessel $>3.0 \mathrm{~mm}, 3.0-2.5$ $\mathrm{mm}$, and $<2.5 \mathrm{~mm}$. Catheter Cardiovasc Interv 2001;53:314322.

8. Süselbeck T, Latsch A, Siri H, et al. Role of vessel size as a predictor for the occurrence of in-stent restenosis in patients with diabetes mellitus. Am J Cardiol 2001;88:243-247.

9. Airoldi F, Colombo A, Morici N, et al. Incidence and predictors of drug-eluting stent thrombosis during and after discontinuation of thienopyridine treatment. Circulation 2007;116:745754.

10. Koh AS, Chia S, Choi LM, et al. Long-term outcomes after coronary bare-metal-stent and drug-eluting-stent implantations: A 'real-world' comparison among patients with diabetes with diffuse small vessel coronary artery disease. Coron Artery Dis 2011;22(1):96-99.
11. Costa MA, Sabate M, Staico R, et al. Anatomical and physiologic assessments in patients with small coronary artery disease: Final results of the Physiologic and Anatomical Evaluation Prior to and After Stent Implantation in Small Coronary Vessels (PHANTOM) trial. Am Heart J 2007;153(2):296.e1e7.

12. O'Connor NJ, Morton JR, Birkmeyer JD, et al. Effect of coronary artery diameter in patients undergoing coronary bypass surgery. Circulation 1996;93:652-655.

13. Cannon LA, Kereiakes DJ, Mann T, et al. A prospective evaluation of the safety and efficacy of TAXUS Element paclitaxeleluting coronary stent implantation for the treatment of de novo coronary artery lesions in small vessels: The PERSEUS Small Vessel trial. EuroIntervention 2011;6(8):920-927.

14. Ito H, Hermiller JB. Percutaneous coronary intervention for small-vessel coronary disease: Highlight on the everolimuseluting stent. Expert Rev Cardiovasc Ther 2010;8(9):12391245.

15. Ortolani P, Ardissino D, Cavallini C, et al. Effect of sirolimuseluting stent in diabetic patients with small coronary arteries (a SES-SMART substudy). Am J Cardiol 2005;96(10):13931398.

16. Qiao SB, Hou Q, Xu B, et al. Comparison of drug-eluting stent and bare-mental stent in the complex small vessel intervention. Chin Med J (Engl) 2006;119(7):596-600.

17. Devito FS, Sousa AG, Feres F, et al. Comparative analysis of intimal hyperplasia after sirolimus-eluting stent and thin-strut bare-metal stent implantation in small coronary arteries. Arq Bras Cardiol 2006;86(4):268-275.

18. Ardissino D, Cavallini C, Bramucci E, et al. Sirolimus-eluting vs. uncoated stents for prevention of restenosis in small coronary arteries: A randomized trial. JAMA 2004;292:2727-2734.

19. Jabara R, Gradman M, Chen zjp, et al. Clinical and angiographic features of small vessel stenting in the drug-eluting stent era. Clin Cardiol 2009;32(6): $40-\mathrm{e} 45$.

20. Tanimoto S, Daemen J, Tsuchida K, et al. Two-year clinical outcome after coronary stenting of small vessels using $2.25 \mathrm{~mm}$ sirolimus and paclitaxel-eluting stents: Insight into the RESEARCH and T-SEARCH registries. Catheter Cardiovasc Interv 2007;69(1):94-103.

21. Holmes DR Jr, Kereiakes DJ. The approach to small vessels in the era of drug-eluting stents. Rev Cardiovasc Med 2005;6(Suppl 1):S31-S37.

22. Moses JW, Nikolsky E, Mehran R, et al. Safety and efficacy of the $2.25 \mathrm{~mm}$ sirolimus-eluting Bx Velocity stent in the treatment of patients with de novo native coronary artery lesions: The SIRIUS 2.25 trial. Am J Cardiol 2006;98:1455-1460.

23. Meier B, Sousa E, Guagliumi G, et al. Sirolimus-eluting coronary stents in small vessels. Am Heart J 2006;151(5):1019. e1-e7.

24. Hermiller JB, Fergus T, Pierson W, et al. Clinical and angiographic comparison of everolimus-eluting and paclitaxeleluting stents in small coronary arteries: A post hoc analysis of the SPIRIT III randomized trial. Am Heart J 2009;158(6):1005-1010.

25. Mauri L, Orav EJ, O'Malley AJ, et al. Relationship of late loss in lumen diameter to coronary restenosis in sirolimus-eluting stents. Circulation 2005;111:321-327.

26. Elezi S, Kastrati A, Neumann FJ, et al. Vessel size and long-term outcome after coronary stent placement. Circulation 1998;98:1875-1880.

27. Urban P, Abizaid A, Banning A, et al. Stent thrombosis and bleeding complications after implantation of sirolimus-eluting coronary stents in an unselected worldwide population. A report from the-SELECT (multi-center post-market surveillance) registry. J Am Coll Cardiol 2011;57(13):1445-1454. 
28. Worthley SG, Abizaid A, Banning A, et al. One-year clinical outcomes after sirolimus-eluting coronary stent implantation for acute myocardial infarction in the worldwide eSELECT registry. J Interv Cardiol 2012 Jun; 25(3):253-61, doi: 10.1111/j.1540-8183.2011.00705.x. Epub 2012 Feb 20.

29. Abizaid A, Costa JR Jr, Banning A, et al. The sirolimus-eluting Cypher Select coronary stent for the treatment of bare-metal and drug-eluting stent restenosis: Insights from the e-SELECT (Multicenter Post-Market Surveillance) registry. JACC Cardiovasc Interv 2012;5(1):64-71.

30. Cuculi F, Banning AP, Abizaid A, et al. Outcomes in patients undergoing multivessel percutaneous coronary intervention using sirolimus-eluting stents: A report from the e-SELECT registry. Euro Intervention 2011;7(8):962-968.

31. Hong YJ, Jeong MH, Abizaid A, et al. Sirolimus-eluting coronary stents in octogenarians: A 1-year analysis of the worldwide e-SELECT Registry. JACC Cardiovasc Interv 2011;4(9):982991.

32. Cutlip DE, Windecker S, Mehran R, et al. Clinical end points in coronary stent trials: A case for standardized definitions. Circulation 2007;115:2344-2351.

33. Montalescot G, White HD, Gallo R, et al. Enoxaparin versus unfractionated heparin in elective percutaneous coronary intervention. N Engl J Med 2006;355:1006-1017.

34. Charlson ME, Pompei P, Ales KL, MacKenzie CR. A new method of classifying prognostic comorbidity in longitudinal studies: Development and validation. J Chronic Dis 1987;40:373-383.

35. Biondi-Zoccai GG, Sangiorgi GM, Antoniucci D, et al. Taxus in Real-life Usage Evaluation Study. Testing prospectively the effectiveness and safety of paclitaxel-eluting stents in over 1000 very high-risk patients: Design, baseline characteristics, procedural data and in-hospital outcomes of the multicenter Taxus in Real-life Usage Evaluation (TRUE) Study. Int J Cardiol 2007;117:349-354.

36. Elezi S, Dibra A, Mehilli J, et al. Vessel size and outcome after coronary drug-eluting stent placement: Results from a large cohort of patients treated with sirolimus- or paclitaxel-eluting stents. J Am Coll Cardiol 2006;48(7):1304-1309.

37. De Luca G, Suryapranata H, van't Hof AW, et al. Comparison between stenting and balloon angioplasty in patients undergoing primary angioplasty of small coronary vessels. Am Heart J 2006;152(5):915-920.

38. Togni M, Eber S, Widmer J, et al. Impact of vessel size on outcome after implantation of sirolimus-eluting and paclitaxeleluting stents: A subgroup analysis of the SIRTAX trial. J Am Coll Cardiol 2007;50(12):1123-1131.

39. Kastrati A, Dibra A, Mehilli J, et al. Predictive factors of restenosis after coronary implantation of sirolimus or paclitaxel eluting stents. Circulation 2006;113(19):2293-2300.

40. Rodriguez-Granillo GA, Valgimigli M, Garcia-Garcia HM, et al. One-year clinical outcome after coronary stenting of very small vessels using $2.25 \mathrm{~mm}$ sirolimus and paclitaxel eluting stents: A comparison between the RESEARCH and T-SEARCH registries. J Invasive Cardiol 2005;17(8):409-412.

41. Seabra-Gomes R, Sousa JE, Sousa A, et al. Small coronary arteries treated with sirolimus eluting stents: One-year results of the PORTO multicentre registry. Euro Intervention 2007;3(2):197-205

42. Mehilli J, Dibra A, Kastrati A, et al. Randomized trial of paclitaxel and sirolimus eluting stents in small coronary arteries. Eur Heart J 2006;27:260-266.

43. Li JJ, Xu B, Yang YJ, et al. Drug-eluting stent for the treatment of small coronary lesion: Comparison between sirolimus and paclitaxel eluting stent. Chin Med J (Engl) 2007;120(7):569573.

44. Nikolsky E, Moses JW, Mahran R, et al. Safety and efficacy of the 2.25-mm sirolimus-eluting BX Velocity stent in the treatment of patients with de novo native coronary artery lesions: the SIRIUS 2.25 trial. Am J Cardiol 2006;98:14551460.

45. Bucalo R, Capranzano P, Conti G, et al. Sirolimus versus paclitaxel eluting stents in small coronary vessels: Long term outcomes from a single-center registry. J Cardiovasc Med 2010;11(5):365-368

46. Ferrer Garcia MC, Moreno R, Pérez Vizcayno MJ, et al. Failure in the implantation of drug-eluting stents. Frequency and related factors. Med Intensiva 2007;31:423-427.

47. Stone GW, Ellis SG, Cannon L, et al. Comparison of a polymerbased paclitaxel-eluting stent with a bare metal stent in patients with complex coronary artery disease: A randomized controlled trial. JAMA 2005;294:1215-1223.

48. Schofer J, Schlüter M, Gershlick AH, et al. Sirolimus eluting stents for treatment of patients with long atherosclerotic lesions in small coronary arteries: Double-blind, randomised controlled trial (E-SIRIUS). Lancet 2003;362(9390):10931099.

49. Schampaert E, Cohen EA, Schlüter M, et al. The Canadian study of the sirolimus-eluting stent in the treatment of patients with long de novo lesions in small native coronary arteries (C-SIRIUS). J Am Coll Cardiol 2004:43(6):1110-1115.

50. Kaneda H, Ako J, Terashima M, et al. Sirolimus eluting stent implantation in small coronary arteries: A three dimensional intravascular ultrasound study from the SIRIUS trial. Int J Cardiol 2010;138(2):126-130.

51. Unverdorben M, Kleber FX, Heuer H, et al. Treatment of small coronary arteries with a paclitaxel coated balloon catheter. Clin Res Cardiol 2010;99(3):165-174.

52. Huang P, Levin T, Kabour A, et al. Acute and late outcome after use of $2.5 \mathrm{~mm}$ intracoronary stents in small $(<2.5 \mathrm{~mm})$ coronary arteries. Catheter Cardiovasc Interv 2000;49:121-126.

53. Umeda $\mathrm{H}$, Iwase M, Gochi T, et al. Safety and efficacy of 2.5 $\mathrm{mm}$ sirolimus eluting stent implantation at lower deployment pressures in very small vessels $(<2.5 \mathrm{~mm})$. Coron Artery Dis 2009;20(2):163-168

54. Turco MA, Ormiston JA, Popma JJ, et al. Reduced risk of restenosis in small vessels and reduced risk of myocardial infarction in long lesions with the new thin-strut TAXUS Liberté stent: One-year results from the TAXUS ATLAS program. JACC Cardiovasc Interv 2008;1(6):699-709.

55. Hausleiter J, Kastrati A, Mehilli J, et al. Predictive factors for early cardiac events and angiographic restenosis after coronary stent placement in small coronary arteries. J Am Coll Cardiol 2001;40:882-889.

56. Raisuke I, Yuji I, Akiyoshi M, et al. Predictors of restenosis after implantation of $2.5 \mathrm{~mm}$ stents in small coronary arteries. Circ J 2004;68(3):236-240

57. Iijima R, Ikari Y, Wada M, et al. Cutting balloon angioplasty is superior to balloon angioplasty or stent implantation for small coronary artery disease. Coron Artery Dis 2004;15(7):435440.

58. Lee CW, Suh J, Lee SW, et al. Factors predictive of cardiac events and restenosis after sirolimus-eluting stent implantation in small coronary arteries. Catheter Cardiovasc Interv 2007;69(6):821-825.

59. Jeremias A, Sylvia B, Bridges J, et al. Stent thrombosis after successful sirolimus eluting stent implantation. Circulation 2004;109(16):1930-1932. 